\title{
Tratamiento de la coledocolitiasis mediante CPRE e instrumentación a través de un tubo en T
}

\author{
Treatment of choledocholithiasis by ERCP, and instrumentation \\ through a T-tube
}

\author{
Robin Germán Prieto-Ortiz, MD,,$^{*}$ (1) Arturo Iván Duarte-Osorio, MD, ${ }^{2}$ (1) Pablo García-Echeverri, MD, ${ }^{3}$ (B]
}

Hernán Ballén-Párraga, MD. 4 [D

\begin{abstract}
GacCeso abierto
Citación:

Prieto-Ortiz RG, Duarte-Osorio Al, García-

Echeverri P, Ballén-Párraga H. Tratamiento de la

coledocolitiasis mediante CPRE e instrumentación a través de un tubo en T. Rev Colomb

Gastroenterol. 2020;35(3):382-389. https://doi.

org/10.22516/25007440.385

Gastroenterólogo, Hospital Central de la Policía Bogotá, Colombia.

Cirujano general, Hospital Central de la Policía; Bogotá, Colombia.

3 Cirujano general, Hospital Central de la Policía; Bogotá, Colombia.

4 Jefe del Servicio de Gastroenterología, Hospital Central de la Policía; Bogotá, Colombia.

*Correspondencia: Robin Germán Prieto-Ortiz, MD rgprietoo@hotmail.com
\end{abstract}

Fecha recibido: $\quad 02 / 04 / 19$ Fecha aceptado: 05/07/19

\section{Resumen}

El tratamiento de la coledocolitiasis ha evolucionado de forma significativa desde que Robert Abbe realizó la primera coledocotomía y la exploración de las vías biliares en Nueva York, en 1889. La colangiopancreatografía retrógrada endoscópica (CPRE), que inicialmente fue un método diagnóstico, ahora solo tiene validez como método terapéutico. En la actualidad, los principales métodos diagnósticos son la colangioresonancia magnética (CRM) y la ultrasonografía endoscópica (USE). El tratamiento de la coledocolitiasis pasó de la técnica quirúrgica abierta -en la que, de forma rutinaria, se realizaba la coledocorrafia sobre un tubo de Kehr o tubo en T- a la endoscópica, mediante el uso de la CPRE, la esfinteroplastia y la instrumentación con balones y canastilla. Hoy en día se dispone de técnicas adicionales como la litotricia mecánica (LM) o extracorpórea, la dilatación con balón (DB) de gran tamaño y el Spyglass ${ }^{\circledR}$. La técnica laparoscópica se usa desde hace varios años, en diversas partes del mundo, para el tratamiento de la coledocolitiasis. Estudios recientes proponen incluso el cierre primario del colédoco o la coledocoduodenostomía, con lo cual no sería necesaria la utilización del tubo en T. Pero en muchos otros sitios, y por diversas razones, se continúa usando la exploración quirúrgica abierta y el tubo en $\mathrm{T}$, que representa una importante opción en el tratamiento de algunos pacientes. Caso clínico: paciente masculino de 88 años, con coledocolitiasis recidivante, cálculo gigante de difícil manejo endoscópico y sepsis de origen biliar, que requirió drenaje quirúrgico abierto de urgencias. Se realizó una coledocotomía, y se dejó el tubo en T. Posteriormente, se efectuó un tratamiento exitoso conjunto, mediante instrumentación por el tubo en T, por parte de cirugía general, y CPRE, por gastroenterología.

\section{Palabras clave}

Coledocolitiasis, colangiopancreatografía retrógrada endoscópica (CPRE), instrumentación de la vía biliar, tubo de Kehr, tubo en T, laparoscopia

\begin{abstract}
The treatment of choledocholithiasis has evolved significantly since Robert Abbé performed the first bile duct exploration via choledochotomy in New York in 1889. Endoscopic retrograde cholangiopancreatography (ERCP), which was initially used for diagnosis, is now only valid as a therapeutic tool. Currently, the main diagnostic methods are magnetic resonance cholangiopancreatography (MRCP) and endoscopic ultrasound (EUS). The treatment of choledocholithiasis moved from the open surgery in which biliary stenting was routinely performed on a Kehr tube or T-tube, to the endoscopic technique using ERCP, sphincteroplasty and instrumentation with balloons and baskets. Additional techniques are now available such as mechanical or extra-corporeal lithotripsy, endoscopic papillary large balloon dilation and SpyGlass cholangioscopy. The laparoscopic technique has been used for several years in different parts of the world for the treatment of choledocholithiasis. Recent studies even propose performing the primary closure of the bile duct or choledochoduodenostomy, so that the T-tube is not necessary. However, in many other places, and for a variety of reasons, open exploratory surgery and the T-tube continue to be used, being an important option in the treatment of some patients. Case presentation: 88-year-old male patient with recurrent choledocholithiasis and a giant gallstone that was difficult to treat endoscopically, with sepsis of biliary origin, which required open surgical drainage at the emergency room. Choledocotomy was performed, and a T-tube was inserted at the site. Subsequently, a successful joint treatment was performed by the General Surgery Service and the Gastroenterology Service, using T-tube instrumentation and ERCP, respectively.
\end{abstract}

\section{Keywords}

Choledocholithiasis; Endoscopic Retrograde Cholangiopancreatography (ERCP); Bile duct exploration; Kehr tube; Laparoscopic T-tube. 


\section{INTRODUCCIÓN}

Los pacientes que presentan litiasis vesicular y coledociana pueden ser tratados en dos tiempos: mediante la realización de una colangiopancreatografía retrógrada endoscópica (CPRE) y, posteriormente, con colecistectomía abierta o laparoscópica. También pueden ser manejados en un solo tiempo, mediante la realización de una colecistectomía laparoscópica (COLELAP) y la exploración laparoscópica de la vía biliar (ELVB).

Diversos metaanálisis concluyen que, tanto el uso de dos tiempos como el de uno solo, son igualmente efectivos en cuanto a la tasa de éxito, y presentan tasas similares de morbilidad y mortalidad $(1,2)$. El uso de la técnica de uno o de dos tiempos depende de los recursos disponibles y de la experiencia de cada grupo médico.

La cirugía abierta de vesícula, así como la exploración quirúrgica abierta de las vías biliares, sigue realizándose en diversas instituciones de nuestro país, dada la carencia de recursos o de entrenamiento. En los principales centros médicos, su práctica se limita a los pacientes en quienes, por diversas causas, no se puede efectuar el tratamiento endoscópico de la coledocolitiasis, o en quienes la técnica endoscópica no ha sido exitosa.

En los pacientes que presentan coledocolitiasis, la CPRE es considerada en la actualidad el tratamiento de primera elección. Entre tanto, la exploración quirúrgica se reserva para los pacientes en quienes no se ha logrado resolver la coledocolitiasis, dadas las características de los cálculos (tamaño o localización), o debido a las variaciones anatómicas relacionadas o no con antecedentes quirúrgicos, las cuales impiden la adecuada canulación de la vía biliar (3).

El tubo de Kehr (o tubo en T) fue de gran utilidad y de uso común durante muchos años, fundamentalmente durante la última mitad del siglo pasado. Estaba indicado en los pacientes en quienes se realizaba una coledocotomía para exploración exitosa o no de las vías biliares, o como elemento de ferulización en casos de reconstrucción de la vía biliar. Debido a la disponibilidad de la CPRE, su utilización ha disminuido durante los últimos dos decenios $(4,5)$.

En la actualidad, algunos autores proponen el cierre primario del colédoco, así como la coledocorrafia sobre endoprótesis e incluso la coledocoduodenostomía, con lo cual se puede pensar que el uso del tubo en $\mathrm{T}$ tiende a desaparecer (6).

Consideramos que el tubo en $\mathrm{T}$ sigue siendo de gran utilidad en nuestro medio y, a escala mundial, en algunos escenarios seleccionados. Se presenta el caso de un paciente con coledocolitiasis de difícil manejo, en quien se realizó la instrumentación conjunta de la vía biliar, a través de un tubo en $\mathrm{T}$, complementada con duodenoscopia y CPRE, lo que permitió lograr un tratamiento exitoso.

\section{PRESENTACIÓN DEL CASO}

El 31 de diciembre de 2018 ingresó un paciente masculino de 88 años al servicio de urgencias del Hospital Central de la Policía (HOCEN). El hombre presentaba 3 días de evolución de dolor abdominal tipo cólico en el cuadrante superior derecho, asociado a coluria y a malestar general, sin acolia u otros síntomas relacionados. El sujeto refirió como antecedentes de importancia hipertensión arterial tratada con losartán, enfermedad pulmonar obstructiva crónica (EPOC) y colecistectomía 17 años atrás.

En el momento del examen físico de ingreso, se encontró un paciente en condiciones generales aceptables para la edad: tensión arterial (TA) de 128/78 $\mathrm{mm} \mathrm{Hg}$, frecuencia cardíaca (FC) de $60 \mathrm{lpm}$ y frecuencia respiratoria (FR) de $18 \mathrm{rpm}$. Además, el individuo se mostró alerta, afebril, hidratado y sin ictericia mucocutánea, con una disminución bibasal de los ruidos respiratorios, sin signos de irritación peritoneal y con un leve dolor a la palpación del hipocondrio derecho (HCD).

Los paraclínicos de ingreso informaron leucocitos de 13 550, hematocrito del $34 \%$ y hemoglobina de $11 \mathrm{~g} / \mathrm{dL}$. Además, se observaron plaquetas de $532000 / \mathrm{mL}$, fosfatasa alcalina de $977 \mathrm{UI} / \mathrm{L}$, bilirrubina total de $2,22 \mathrm{mg} / \mathrm{dL}$, bilirrubina directa de $1,64 \mathrm{mg} / \mathrm{dL}$ y bilirrubina indirecta de $0,58 \mathrm{mg} / \mathrm{dL}$. La transaminasa glutámico-pirúvica fue de 252 UI/L, mientras que la transaminasa glutámico-oxalacética fue de 220 UI/L. Se realizó una ecografía abdominal total, que reportó un estado de poscolecistectomía e hígado graso.

Así pues, el paciente fue valorado por el servicio de gastroenterología, con sospecha de coledocolitiasis. Por tanto, se solicitó una colangiorresonancia magnética (CRM), cuyo resultado (enero 2 de 2019) describió la presencia de 2 cálculos facetados de 16 y $18 \mathrm{~mm}$, con una dilatación del colédoco distal de hasta $17 \mathrm{~mm}$ y una leve dilatación de la vía biliar intrahepática (Figura 1).

El 3 de enero, el paciente fue conducido a una CPRE (sumada a una papilotomía), realizada por 2 gastroenterólogos con experiencia en la vía biliar. Se observó un hallazgo colangiográfico de 2 cálculos de aproximadamente $20 \mathrm{~mm}$, que no se lograron extraer; tampoco fue posible insertar una endoprótesis (stent). Durante el procedimiento, el hombre presentó inestabilidad hemodinámica, por lo que fue trasladado a la UCI, en donde requirió multisoporte. Dado el diagnóstico de sepsis de origen biliar, se solicitó la valoración de cirugía general.

Con la necesidad de un drenaje de urgencias de la vía biliar, el paciente fue llevado a salas de cirugía y se le realizó un drenaje quirúrgico abierto. Debido a la inestabilidad hemodinámica, solo se efectuó una coledocotomía e instrumentación con pinzas Randall. Se obtuvo un hallazgo intraoperatorio de vía biliar dilatada $(25 \mathrm{~mm})$ y la extrac- 
ción de un cálculo facetado de $20 \mathrm{~mm}$. Además, se realizó un lavado de la vía biliar mediante sonda de Nélaton, la cual avanzó fácilmente hacia el duodeno. Por la celeridad del procedimiento, no se realizó una colangiografía y, finalmente, se practicó una coledocorrafia sobre tubo en $\mathrm{T}$.

Durante el posoperatorio, el paciente permaneció en la UCI por varios días, con soporte inotrópico y respiratorio. Resuelta la emergencia, y ante la buena evolución, se realizó una colangiografía por el tubo en T (enero 9 de 2019), la cual identificó un defecto de llenado en la porción distal del colédoco, que alcanzó un diámetro de $18 \mathrm{~mm}$, con defecto de llenado, que sugirió la presencia de un cálculo. No se evidenció el paso del medio de contraste hacia las asas intes- tinales. Se determinó un diagnóstico de síndrome biliar, al parecer, secundario a coledocolitiasis (Figura 2).

Se decidió, entonces, realizar de forma conjunta la instrumentación de la vía biliar, efectuada por los servicios de cirugía general, a través del tubo en $\mathrm{T}$, y por el servicio de gastroenterología, mediante vía endoscópica. El procedimiento se llevó a cabo en la sala de fluoroscopia, para lo cual se avanzó una canastilla de Dormia y un litotriptor mecánico por el tubo en T. En consecuencia, se logró la fragmentación parcial y el avance de fragmentos al duodeno.

Asimismo, por vía endoscópica, se completó la fragmentación y la extracción de los cálculos, mediante el uso de una canastilla de Dormia (Figura 3) y de un balón extractor.

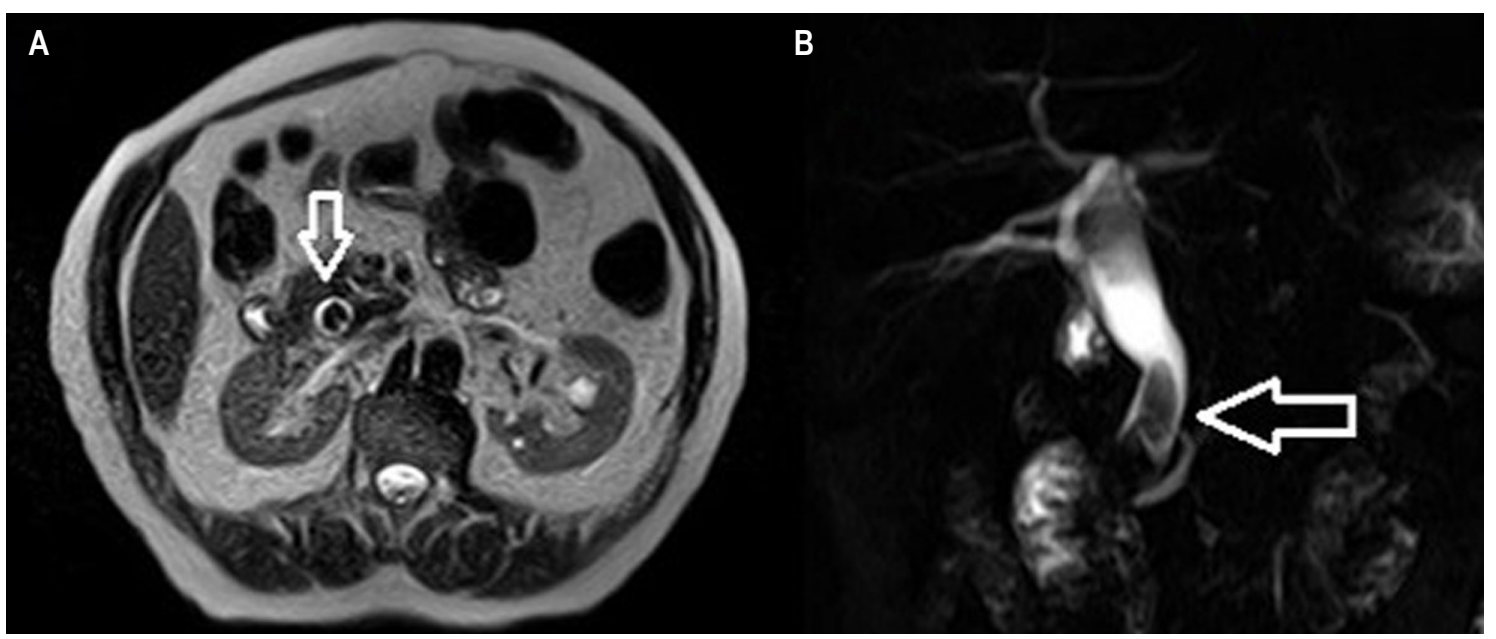

Figura 1. CRM. A. Corte coronal en el que se observa el cálculo de gran tamaño, que ocupa completamente la luz el colédoco. B. Reconstrucción tridimensional en la cual se observan los 2 cálculos facetados y la dilatación del colédoco.
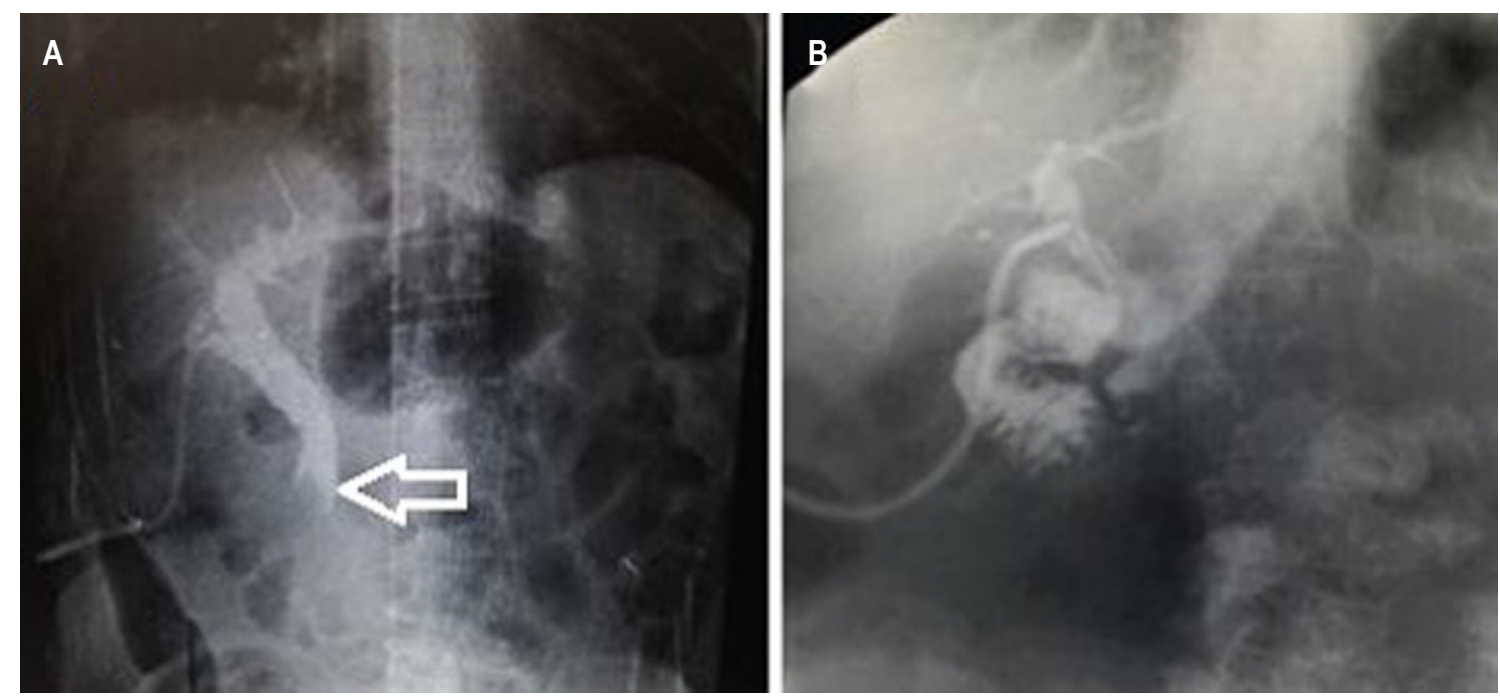

Figura 2. A. Se observa la dilatación de la vía biliar y el cálculo gigante que impide el paso del medio al duodeno. B. Colangiografía luego de la instrumentación conjunta, sin que se encuentren los defectos de llenado ni el adecuado paso del medio al duodeno. 


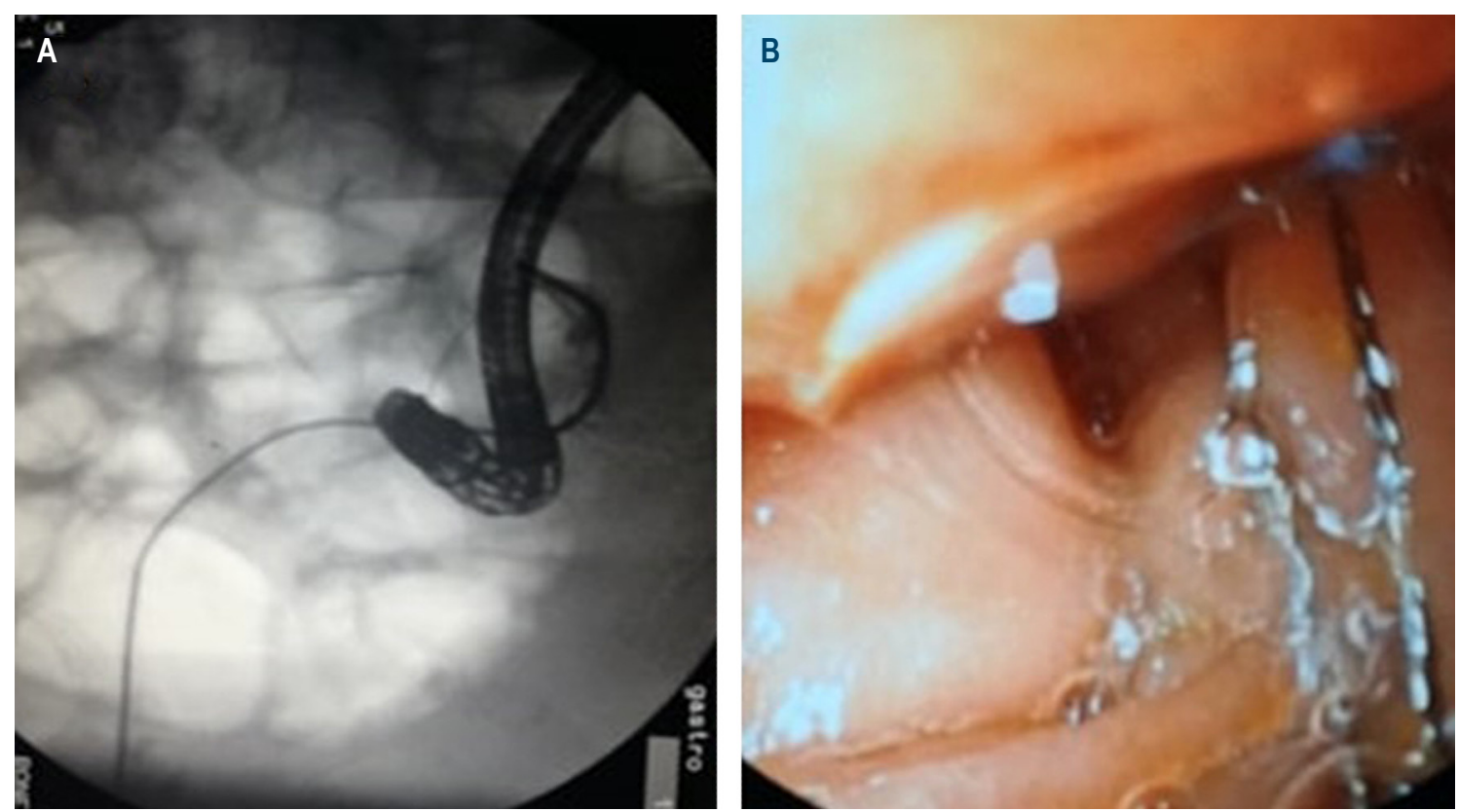

Figura 3. A. Fluoroscopia de la instrumentación conjunta. B. Visión duodenoscópica de la canastilla de Dormia avanzada por el tubo en $\mathrm{T}$.

Se realizó una colangiografía por el tubo en T y se observó un adecuado paso del medio de contraste al duodeno, sin extravasaciones de este, y sin evidencia de cálculos residuales (Figura 2B).

El paciente evolucionó de forma adecuada y, antes del egreso, se realizó una colangiografía por el tubo en T, así como un perfil hepático, cuyos resultados fueron completamente normales.

\section{DISCUSIÓN}

La colelitiasis es una enfermedad endémica que afecta hasta el $20 \%$ de la población, tanto en países orientales como occidentales $(7,8)$. Entre el 10-20\% de los pacientes con esta patología pueden presentar coledocolitiasis de forma simultánea. Cerca de la mitad de las personas con colelitiasis pueden ser asintomáticas, sin requerir cirugía, pero, en aquellas con coledocolitiasis, el tratamiento es mandatorio, dado el riesgo de pancreatitis aguda o de colangitis por obstrucción del flujo pancreático o biliar (9).

Durante muchos años, el diagnóstico de la coledocolitiasis se confirmó mediante la CPRE. Sin embargo, en la actualidad este diagnóstico es relativamente fácil y puede confirmarse mediante la CRM o por USE. En la gran mayoría de los casos, la coledocolitiasis es secundaria a la migración de cálculos desde la vesícula, y en muy pocos se debe a la formación primaria o de novo de cálculos en la vía biliar (7).
La primera coledocotomía, así como la exploración de las vías biliares, se atribuye a Robert Abbe, desarrollada en Nueva York en 1889 (10). Desde ese entonces, y por cerca de un siglo, el tratamiento de la colecistocoledocolitiasis se realizó en un solo tiempo quirúrgico, mediante la cirugía abierta. Durante los primeros años de la COLELAP, en la década de los 80 , la imposibilidad de la exploración laparoscópica de la vía biliar favoreció la realización de la CPRE. Por tanto, esta se convirtió en la técnica estándar de la actualidad, y el tratamiento de la colecistocoledocolitiasis pasó de uno a dos tiempos.

Aunque la tasa de éxito es tan alta (87-97\%), entre el 5 y el $10 \%$ de los pacientes requieren de 2 o más CPRE (1,2, 7). Cuando, pese a ello, no se logra la resolución de la coledocolitiasis, se debe realizar una exploración quirúrgica, laparoscópica o abierta.

En la actualidad, el tratamiento de la coledocolitiasis, bien sea primaria o secundaria, puede realizarse mediante cirugía abierta o laparoscópica (simultánea o en un tiempo en los casos de colelitiasis) y por técnicas endoscópicas o combinadas con técnicas quirúrgicas en los casos de coledocolitiasis (secuencial o en dos tiempos) (11).

En un metaanálisis de 16 estudios aleatorizados, en el cual se compararon la cirugía abierta, la cirugía laparoscópica y las técnicas combinadas con endoscopia (COLELAP más CPRE) para el tratamiento de la coledocolitiasis, no se encontró ninguna diferencia significativa en cuanto 
a la morbilidad (13-20\%) y a la mortalidad (0-3\%). Sin embargo, en los grupos de cirugía abierta se identificó un menor porcentaje de cálculos persistentes (6\%), en comparación con los grupos de COLELAP y CPRE (16\%).

A partir de estos resultados, se concluyó que la técnica abierta pareció ser superior a la combinada en el tratamiento de la coledocolitiasis, aunque la primera se asoció con una mayor estancia hospitalaria $(9,12)$. La técnica laparoscópica presentó la ventaja de ser más costoefectiva y sin demostrar la morbimortalidad asociada a la CPRE (13).

Entre tanto, debido a la mejora en los materiales y en la técnica laparoscópica, desde hace ya varios años se está realizando en muchos países la ELVB. Con esta técnica, se resuelve en un solo tiempo la colecistocoledocolitiasis, con todos los beneficios de la laparoscopia y sin los inconvenientes relacionados con la CPRE (14).

La CPRE y los métodos laparoscópicos de exploración de la vía biliar no se encuentran disponibles en muchos hospitales, debido a la falta de equipos o de personal médico entrenado. La exploración de la vía biliar se debe realizar según los recursos y la experiencia del personal disponible en cada institución, y aunque la cirugía abierta es un método invasivo, no se debe considerar obsoleta, dado que aún es de gran utilidad en casos complejos o en aquellos que requieren conversión a técnica abierta $(13,14)$.

Una vez resuelta la coledocolitiasis, la sutura del colédoco (o coledocorrafia) se puede realizar como tradicionalmente se hace en la cirugía abierta, insertando un tubo en T en la vía biliar a través de la incisión en el colédoco. Esto permite ferulizar la vía biliar y lograr una sutura segura, a fin de impedir la fuga de bilis o la formación de colecciones intraabdominales, ya que se favorece la formación de una fístula. Además, permite descomprimir la vía biliar si se da un aumento de la presión en el esfínter de la papila duodenal y, en caso de cálculos residuales, permite la reexploración de la vía biliar, lo que evita una nueva CPRE o una reintervención quirúrgica $(15,16)$.

En principio, la colocación del tubo en $\mathrm{T}$ fue una parte imprescindible de la técnica laparoscópica, pero estudios recientes han demostrado que la coledocorrafia sobre una prótesis biliar transpapilar, por vía laparoscópica, es un método efectivo y seguro para el tratamiento de la colecistocoledocolitiasis en un único tiempo. De esta forma, se evitan todas las complicaciones relacionadas con el manejo del tubo en $\mathrm{T}$ y se logra una menor estancia hospitalaria. En efecto, en la actualidad algunos autores consideran que el tubo en $\mathrm{T}$ solo se debe usar en los casos en que técnicamente no sea posible la colocación de la prótesis biliar (17).

Entre tanto, otros autores proponen realizar el cierre laparoscópico primario de la vía biliar, basados en un metaanálisis en el que se revisaron 12 estudios. Este trabajo develó que el uso rutinario del tubo en $\mathrm{T}$ no se justifica, dada la morbilidad relacionada con su utilización, en comparación con la muy baja morbilidad presentada en los pacientes sometidos al cierre primario $(18,19)$.

Así, la exploración de la vía biliar y la realización de la coledocoduodenostomía laparoscópica, efectuada por grupos experimentados, ha demostrado ser eficaz y segura en el tratamiento de casos complejos de coledocolitiasis (coledocolitiasis múltiple, cálculos impactados, cálculos en conductos hepáticos, estenosis biliares y coledocolitiasis recidivante) o en escenarios de una CPRE fallida. Esta técnica quirúrgica ha demostrado, además, una corta estancia hospitalaria (20).

En pacientes con sepsis de origen biliar e inestabilidad hemodinámica, el tratamiento de urgencia consiste en drenar la vía biliar, lo cual puede realizarse en forma percutánea, endoscópica (CPRE) o quirúrgica, así como por vía laparoscópica o abierta, como ocurrió en el caso de nuestro paciente.

\section{Técnica endoscópica: CPRE}

La CPRE posoperatoria está indicada en los casos en los que la ELVB no ha sido exitosa, o cuando, luego de la cirugía, se identifica la presencia de cálculos mediante la colangiografía por el tubo en T. Esto puede ocurrir hasta en el 2,5\% de los pacientes (21).

El 85-90 \% de los cálculos de la vía biliar se pueden tratar exitosamente mediante la técnica endoscópica estándar, con la realización de una esfinterotomía, el uso del balón y la utilización de una canastilla de Dormia (22).

La esfinterotomía endoscópica (EE) de la papila fue descrita de forma simultánea en 1974 tanto por Classen y Demling, en Alemania, como por Kaway, en Japón. Esta técnica consiste en realizar un corte en el esfínter de Oddi, a fin de permitir la extracción de cálculos mediante el arrastre con canastillas o balones $(23,24)$.

Así pues, las causas principales de una CPRE fallida son los cálculos de gran tamaño $(>2 \mathrm{~cm}$.), la impactación de estos y la estreches distal del colédoco. En tales casos, es necesario el uso de técnicas adicionales $(22,25)$.

\section{Técnica de rendezvous}

La técnica laparoscópica de rendezvous fue descrita por primera vez en 1998 (26). Sin embargo, el primer tratamiento combinado de instrumentación quirúrgica y endoscópica de la vía biliar fue obra del Dr. Rodolfo Mazzariello. En 1976, él, junto al Dr. Magnanini, realizaron en Buenos Aires (Argentina) una papilotomía endoscópica, que consistió en hacer avanzar un papilótomo por la fístula de un tubo en T. El papilótomo fue recuperado y ubicado mediante una endoscopia, con el fin de realizar el corte eléctrico, y luego de ello se logró la eliminación espontánea del cálculo (27). 
Antes de ese momento, el Dr. Mazzariello había descrito la técnica que lleva su nombre y que fue utilizada por muchos cirujanos. En ella se realiza la exploración y la extracción de los cálculos de la vía biliar, a través del tubo en $\mathrm{T}$ o de la fístula formada por este (28).

En la literatura se han descrito algunas modificaciones de la técnica de rendezvous. Un ejemplo de estos cambios puede apreciarse en el caso de aquellos pacientes intervenidos quirúrgicamente, que tienen tubo en $\mathrm{T}$ en la vía biliar, y en quienes a través de dicho tubo se hace avanzar la guía que se recupera mediante el endoscopio, para luego realizar la esfinterotomía y la exploración de las vías biliares (29).

De cualquier manera, en el caso presentado en este artículo, la disponibilidad del tubo en $\mathrm{T}$ permitió que, en un paciente con coledocolitiasis de difícil manejo y CPRE previa fallida, se realizara el tratamiento exitoso. Dicho tratamiento se logró mediante la técnica combinada de instrumentación de la vía biliar -con la canastilla de Dormia y el litotriptor a través del tubo en T-y la instrumentación por medio de una CPRE con canastilla de Dormia y balón extractor.

El uso de la colangiografía transhepática percutánea (CTHP) ha sido descrito como parte de una técnica de rendezvous modificada. En ella se hace avanzar la guía a través de la CTHP, para luego ser recuperada en el duodeno y realizar la esfinterotomía $(30,31)$.

\section{CÁLCULOS GIGANTES 0 DIFÍCILES}

Entre el 10-15\% de los pacientes con coledocolitiasis presentan cálculos gigantes (10-15 mm), cuya extracción, aunque difícil, puede resolverse de forma endoscópica, y en la mayoría de los casos, mediante diversas técnicas (32).

La técnica de dilatación con balón (DB) fue descrita por primera vez por Staritz en 1982 (33). Esta consiste en la dilatación del esfínter biliar con balones de hasta $10 \mathrm{~mm}$ de diámetro, sin la realización previa de una esfinterotomía. Su objetivo es reemplazar a la EE para la extracción de cálculos. En 2004, se publicó un estudio aleatorizado en el que se encontró que la incidencia de pancreatitis fue del 15,4\% al realizar la DB, en comparación con el $0,8 \%$ con EE (34).

Algunos metaanálisis publicados posteriormente concluyeron que el uso de la DB para la extracción de cálculos, en comparación con la EE, se asocia a una menor tasa de éxito terapéutico y a una mayor necesidad de litotricia mecánica (LM). Por todos estos motivos, la DB no está recomendada como método rutinario para la extracción de cálculos, pero es una opción razonable en pacientes con coagulopatía, divertículo periampular o antecedente de cirugía tipo Billroth II (35).

Así pues, la LM logra la fragmentación de los cálculos en cerca del $95 \%$ de los casos; en los restantes, la falla se debe a la inadecuada apertura del litotriptor dentro de la vía biliar o a la difícil captura del cálculo, debida a su forma o tamaño (36).

Entre tanto, la esfinterotomía endoscópica más la dilatación con balón de gran tamaño (EE-DBGT) fue descrita por Esroz en 2003 para el tratamiento de cálculos difíciles. Esta técnica ha demostrado lograr un éxito en el 89-95\% de los pacientes. El procedimiento consiste en realizar una esfinterotomía y dilatar el orificio papilar con balones de 12-20 mm de diámetro, lo cual causa una gran dilatación del orificio papilar y facilita la extracción de cálculos grandes o que ofrecen una dificultad inusual $(25,37)$.

En la actualidad, la EE-DBGT es considerada un procedimiento efectivo y seguro para el tratamiento de la coledocolitiasis complicada. Este método disminuye la necesidad de litotricia y de sesiones repetidas de CPRE. La selección apropiada de los pacientes y algunas consideraciones técnicas son importantes para reducir el riesgo de complicaciones (36-39).

Por lo general, los cálculos de gran tamaño o impactados se manejan con técnicas de fragmentación como la LM. En casos fallidos, el tratamiento se da, ya sea mediante litotricia electrohidráulica (LEH), litotricia con ondas de choque extracorpórea (LOCE) (que es de gran ayuda en pacientes con anormalidades anatómicas como aquellos con cirugías Billroth II o Y de Roux), o bien con litotricia láser (LL), la cual es guiada por una colangioscopia convencional.

En años recientes se ha dispuesto del Spyglass ${ }^{\oplus}$, una técnica en la que, mediante una colangioscopia directa, un solo operador puede realizar un procedimiento diagnóstico o terapéutico con LEH o laser en la vía biliar y pancreática. Además, permite tener un mejor campo visual, así como una imagen endoscópica superior. No obstante, presenta el inconveniente del alto costo, lo que limita su uso en la mayoría de las instituciones (22).

\section{CONCLUSIONES}

La cirugía abierta de vesícula, así como la exploración quirúrgica de las vías biliares, siguen siendo una realidad en diversas instituciones de nuestro país, dada la carencia de recursos $y$, en ocasiones, la falta de entrenamiento o experiencia.

En los principales centros médicos, la exploración abierta o laparoscópica de la vía biliar se reserva para aquellos pacientes, en quienes, por diversas causas -y a pesar de la gran variedad de técnicas que actualmente permiten la resolución de la coledocolitiasis-, ha sido imposible la realización de dicho procedimiento.

El uso del tubo en T continúa siendo de gran utilidad en los pacientes que son conducidos a exploración quirúrgica de la vía biliar. En los casos de coledocolitiasis persistente, facilita en gran medida la exploración y la resolución de la coledocolitiasis, de forma conjunta con los métodos endoscópicos. 


\section{Fuentes de financiación}

Los recursos empleados en el desarrollo de este artículo han sido aportados de forma exclusiva por sus autores.

\section{Conflicto de intereses}

Los autores declaran que no existe conflicto de intereses relacionado con la producción o la publicación de este artículo.

\section{Consentimiento informado}

Para la presentación y publicación de este caso, se contó con el consentimiento informado por escrito del paciente y de su familia.

\section{REFERENCIAS}

1. Bansal VK, Misra MC, Rajan K, Kilambi R, Kumar S, Krishna A, Kumar A, Pandav CS, Subramaniam R, Arora MK, Garg PK. Single-stage laparoscopic common bile duct exploration and cholecystectomy versus two-stage endoscopic stone extraction followed by laparoscopic cholecystectomy for patients with concomitant gallbladder stones and common bile duct stones: a randomized controlled trial. Surg Endosc. 2014;28(3):875-85. http://doi.org/10.1007/s00464-013-3237-4

2. Ding G, Cai W, Qin M. Single-stage vs. two-stage management for concomitant gallstones and common bile duct stones: a prospective randomized trial with long-term follow-up. J Gastrointest Surg. 2014;18(5):947-951. http://doi.org/10.1007/s11605-014-2467-7

3. Hungness ES, Soper NJ. Management of common bile duct stones. J Gastrointest Surg. 2006;10(4):612-619. http://doi.org/10.1016/j.gassur.2005.08.015

4. Xiao LK, Xiang JF, Wu K, Fu X, Zheng MY, Song XX, Xie W. The reasonable drainage option after laparoscopic common bile duct exploration for the treatment of choledocholithiasis. Clin Res Hepatol Gastroenterol. 2018;42(6):564-569. http://doi.org/10.1016/j.clinre.2018.07.005

5. Xu Y, Dong C, Ma K, Long F, Jiang K, Shao P, Liang R, Wang L. Spontaneously removed biliary stent drainage versus T-tube drainage after laparoscopic common bile duct exploration. Medicine (Baltimore). 2016;95(39):e5011. http://doi.org/10.1097/MD.0000000000005011

6. Yin Z, Xu K, Sun J, Zhang J, Xiao Z, Wang J, Niu H, Zhao $\mathrm{Q}, \mathrm{Lin}$ S, Li Y. Is the end of the T-tube drainage era in laparoscopic choledochotomy for common bile duct stones is coming? A systematic review and meta-analysis. Ann Surg. 2013;257(1):54-66. http://doi.org/10.1097/SLA.0b013e318268314b

7. Costi R, Gnocchi A, Di Mario F, Sarli L. Diagnosis and management of choledocholithiasis in the golden age of imaging, endoscopy and laparoscopy. World J Gastroenterol. 2014;20(37):13382-13401. http://doi.org/10.3748/wjg.v20.i37.13382

8. Everhart JE, Khare M, Hill M, Maurer KR. Prevalence and ethnic differences in gallbladder disease in the United
States. Gastroenterology. 1999;117(3):632-639. http://doi.org/10.1016/s0016-5085(99)70456-7

9. Dasari BV, Tan CJ, Gurusamy KS, Martin DJ, Kirk G, McKie L, Diamond T, Taylor MA. Surgical versus endoscopic treatment of bile duct stones. Cochrane Database Syst Rev. 2013; (9):CD003327. http://doi.org/10.1002/14651858.CD003327.pub3

10. Verbesey JE, Birkett DH. Common bile duct exploration for choledocholithiasis. Surg Clin North Am. 2008;88(6):1315-28. http://doi.org/10.1016/j.suc.2008.08.002

11. Restrepo C, Sierra S, Penagos S, Palacios OG, Mendoza MC. Manejo sincrónico de la colecistocoledocolitiasis, ¿seguro? Rev Colomb Cir. 2014;29(3):197-203.

12. Clayton ES, Connor S, Alexakis N, Leandros E. Metaanalysis of endoscopy and surgery versus surgery alone for common bile duct stones with the gallbladder in situ. Br J Surg. 2006;93(10):1185-1191. http://doi.org/10.1002/bjs.5568

13. Rogers SJ, Cello JP, Horn JK, Siperstein AE, Schecter WP, Campbell AR, Mackersie RC, Rodas A, Kreuwel HT, Harris HW. Prospective randomized trial of LC+LCBDE vs ERCP/S+LC for common bile duct stone disease. Arch Surg. 2010;145(1):28-33. http://doi.org/10.1001/archsurg.2009.226

14. Berggren U, Gordh T, Grama D, Haglund U, Rastad J, Arvidsson D. Laparoscopic versus open cholecystectomy: hospitalization, sick leave, analgesia and trauma responses. Br J Surg. 1994;81(9):1362-1365. http://doi.org/10.1002/bjs.1800810936

15. Williams JA, Treacy PJ, Sidey P, Worthley CS, Townsend NC, Russell EA. Primary duct closure versus T-tube drainage following exploration of the common bile duct. Aust N Z J Surg. 1994;64(12):823-826. http://doi.org/10.1111/j.1445-2197.1994.tb04556.x

16. Palermo M, Giménez M, Álvarez F, Ortega A, Bruno M, Tarsitano FJ. Abordaje transfistular para el tratamiento de la litiasis residual de la vía biliar. Acta Gastroenterol Latinoam. 2010;40(3):239-243. 
17. Martínez D, Parra P, Díaz D, Lorente JM. Exploración laparoscópica de la vía biliar y coledocorrafia sobre prótesis biliar: dejando atrás el tubo de Kehr. Rev Esp Enferm. 2013;105(3):125-130. http://dx.doi.org/10.4321/S1130-01082013000300002

18. Gurusamy KS, Koti R, Davidson BR. T-tube drainage versus primary closure after laparoscopic common bile duct exploration. Cochrane Database Syst Rev. 2013;(6):CD005641. http://doi.org/10.1002/14651858.CD005641.pub3

19. Liu JG, Wang YJ, Shu GM, Lou C, Zhang J, Du Z. Laparoscopic versus endoscopic management of choledocholithiasis in patients undergoing laparoscopic cholecystectomy: a meta-analysis. J Laparoendosc Adv Surg Tech A. 2014;24(5):287-294. http://doi.org/10.1089/lap.2013.0546

20. Senthilnathan P, Sharma D, Sabnis SC, Srivatsan Gurumurthy S, Senthil Anand E, Nalankilli VP, Anand Vijai N, Praveen Raj P, Parthasarathy R, Rajapandian S, Palanivelu C. Laparoscopic choledochoduodenostomy as a reliable rescue procedure for complicated bile duct stones. Surg Endosc. 2018;32(4):1828-1833. http://doi.org/10.1007/s00464-017-5868-3

21. Anwar S, Rahim R, Agwunobi A, Bancewicz J. The role of ERCP in management of retained bile duct stones after laparoscopic cholecystectomy. $\mathrm{N} Z$ Med J. 2004;117(1203):U1102.

22. Gómez M, Gutiérrez O, Jaramillo M. Manejo del cálculo difícil en la vía biliar: serie de casos. Rev Col Gastroenterol. 2015;30(4):461-468. https://doi.org/10.22516/25007440.10

23. Classen M, Demling L. Endoskopische Sphinkterotomie der Papilla Vateri und Steinextraktion aus dem Ductus choledochus. Dtsch Med Wochenschr 1974;99(11): 496-497. https://doi.org/10.1055/s-0028-1107790

24. Kawai K, Akasaka Y, Murakami K, Tada M, Koli Y. Endoscopic sphincterotomy of the ampulla of Vater. Gastrointest Endosc. 1974;20(4):148-151. http://doi.org/10.1016/s0016-5107(74)73914-1

25. Ersoz G, Tekesin O, Ozutemiz AO, Gunsar F. Biliary sphincterotomy plus dilation with a large balloon for bile duct stones that are difficult to extract. Gastrointest Endosc. 2003;57(2):156-159. http://doi.org/10.1067/mge.2003.52

26. Cavina E, Franceschi M, Sidoti F, Goletti O, Buccianti P, Chiarugi M. Laparo-endoscopic "rendezvous": a new technique in the choledocholithiasis treatment. Hepatogastroenterology. 1998;45(23):1430-1435.

27. Mazzariello R. Tratamiento instrumental postoperatorio de la patología biliar no litiásica. Rev Arg Cir. 1978;34:21-25.

28. Mazzariello RM. Transcholecystic extraction of residual calculi in common bile duct. Surgery. 1974;75(3):338-347.

29. Odabasi M, Yildiz MK, Abuoglu HH, Eris C, Ozkan E, Gunay E, Aktekin A, Muftuoglu MT. A modified Rendezvous ERCP technique in duodenal diverticulum.
World J Gastrointest Endosc. 2013;5(11):568-73. http://doi.org/10.4253/wjge.v5.i11.568

30. Lee JH, Kim HW, Kang DH, Choi CW, Park SB, Kim SH, Jeon UB. Usefulness of percutaneous transhepatic cholangioscopic lithotomy for removal of difficult common bile duct stones. Clin Endosc. 2013;46(1):65-70. http://doi.org/10.5946/ce.2013.46.1.65

31. Meek J, Fletcher S, Crumley K, Culp WC, Meek M. Percutaneous rendezvous technique for the management of a bile duct injury. Radiol Case Rep. 2017;13(1):175-178. http://doi.org/10.1016/j.radcr.2017.11.004

32. Garzón MA, Hernández G, Pérez O, Martínez JD, Molano JC, Marulanda JC, Lizarazo JI, Hormaza N, Rey MH. Factores asociados con el uso de litotripsia mecánica en pacientes con cálculos gigantes de la vía biliar: ¿puede la dilatación papilar con balón grande disminuir su requerimiento? Rev Col Gastroenterol. 2017;32(3):202-208. http://doi.org/10.22516/25007440.151

33. Staritz M, Ewe K, Meyer zum Büschenfelde KH. Endoscopic papillary dilatation: an alternative to papillotomy? Dtsch Med Wochenschr. 1982;107(23):895-897. http://doi.org/10.1055/s-2008-1070041

34. Disario JA, Freeman ML, Bjorkman DJ, Macmathuna P, Petersen BT, Jaffe PE, Morales TG, Hixson LJ, Sherman S, Lehman GA, Jamal MM, Al-Kawas FH, Khandelwal M, Moore JP, Derfus GA, Jamidar PA, Ramirez FC, Ryan ME, Woods KL, Carr-Locke DL, Alder SC. Endoscopic balloon dilation compared with sphincterotomy for extraction of bile duct stones. Gastroenterology. 2004;127(5):1291-9. http://doi.org/10.1053/j.gastro.2004.07.017

35. ASGE Standards of Practice Committee, Maple JT, Ikenberry SO, Anderson MA, Appalaneni V, Decker GA, Early D, Evans JA, Fanelli RD, Fisher D, Fisher L, Fukami N, Hwang JH, Jain R, Jue T, Khan K, Krinsky ML, Malpas P, Ben-Menachem T, Sharaf RN, Dominitz JA. The role of endoscopy in the management of choledocholithiasis. Gastrointest Endosc. 2011;74(4):731-44. http://doi.org/10.1016/j.gie.2011.04.012

36. García VA, Zárate AM, Corral A, Pérez A. Manejo actual de la coledocolitiasis. 1999;62(2):121-127.

37. Jeong SU, Moon SH, Kim MH. Endoscopic papillary balloon dilation: revival of the old technique. World J Gastroenterol. 2013;19(45):8258-8268. http://doi.org/10.3748/wjg.v19.i45.8258

38. Espinoza J, Aliaga M, Rodríguez J, Miraval E, Zegarra A, Bravo E, Prochazka R. Esfinterotomía seguida de dilatación con balón de gran tamaño en el manejo de coledocolitiasis. Eficacia y seguridad en un hospital de Lima-Perú. Rev Gastroenterol Perú. 2016;36(3):203-208.

39. Marín L, Vera A, Gómez A, Cervera Z, Dávalos M, Alva E, Palacios F, Díaz R, Guzmán E, García G. Dilatación con balón de gran diámetro en el manejo de coledocolitiasis de difícil extracción: experiencia clínica. Rev Gastroenterol Perú. 2016;36(4):330-335. 